\title{
ARTICLE Orally consumed cannabinoids provide long-lasting relief of allodynia in a mouse model of chronic neuropathic pain
}

\author{
Antony D. Abraham ${ }^{1}$, Edward J. Y. Leung ${ }^{1}$, Brenden A. Wong ${ }^{1}$, Zeena M. G. Rivera $\mathbb{D}^{1}$, Lauren C. Kruse ${ }^{2}$, Jeremy J. Clark ${ }^{2}$ and \\ Benjamin B. Land ${ }^{1}$
}

Chronic pain affects a significant percentage of the United States population, and available pain medications like opioids have drawbacks that make long-term use untenable. Cannabinoids show promise in the management of pain, but long-term treatment of pain with cannabinoids has been challenging to implement in preclinical models. We developed a voluntary, gelatin oral selfadministration paradigm that allowed male and female mice to consume $\Delta^{9}$-tetrahydrocannabinol, cannabidiol, or morphine ad libitum. Mice stably consumed these gelatins over 3 weeks, with detectable serum levels. Using a real-time gelatin measurement system, we observed that mice consumed gelatin throughout the light and dark cycles, with animals consuming less THC-gelatin than the other gelatin groups. Consumption of all three gelatins reduced measures of allodynia in a chronic, neuropathic sciatic nerve injury model, but tolerance to morphine developed after 1 week while THC or CBD reduced allodynia over three weeks. Hyperalgesia gradually developed after sciatic nerve injury, and by the last day of testing, THC significantly reduced hyperalgesia, with a trend effect of $C B D$, and no effect of morphine. Mouse vocalizations were recorded throughout the experiment, and mice showed a large increase in ultrasonic, broadband clicks after sciatic nerve injury, which was reversed by THC, CBD, and morphine. This study demonstrates that mice voluntarily consume both cannabinoids and opioids via gelatin, and that cannabinoids provide long-term relief of chronic pain states. In addition, ultrasonic clicks may objectively represent mouse pain status and could be integrated into future pain models.

Neuropsychopharmacology (2020) 45:1105-1114; https://doi.org/10.1038/s41386-019-0585-3

\section{INTRODUCTION}

Chronic pain, defined as pain that lasts at least 12 weeks, affects between 40 and 100 million individuals in the United States [1-3], with an annual economic burden of USD 600 billion [4]. The severity of chronic pain can differ, but approximately 11 million adults report major restrictions in activity due to their pain state [3]. Current treatments for chronic pain are limited, and opioids are often prescribed despite high tolerance and addiction risks [5-7]. The large increase in prescriptions for opioid medications indicated for pain over the last 20-30 years has contributed to the current opioid crisis in the United States [8], and thus it is important to identify and validate alternative medications for chronic pain treatment.

Cannabinoids are promising candidates for the treatment of pain $[9,10]$. Numerous studies in preclinical models and human trials have shown that the principal cannabis phytocannabinoids $\Delta^{9}$ tetrahydrocannabinol (THC) and cannabidiol (CBD), as well as synthetic cannabimimetic ligands, are able to relieve various pain states, although the extent to which they provide true pain relief in humans remains controversial $[11,12]$. While many of these studies have focused on acute outcomes of only one or several drug administrations, long-term studies of cannabinoids in humans have shown mixed, and often subtle effects in treatment of pain [11, 13], suggesting that acute pain models may be inadequate when considering chronic pain. To better predict treatment outcomes in humans, we aimed to develop a preclinical model for long-term cannabinoid intake in the context of chronic pain states.

One major challenge to long-term administration of cannabinoids in preclinical models is that most have historically relied on experimenter administration, which does not closely resemble intake patterns of humans in either route of administration, dose, or timing. Efforts have recently been made to model human cannabinoid consumption in rodents more closely, including use of vapor chambers [14, 15], or sweetened, orally consumed solutions [16]. However, in these models, intake was still limited to a relatively short period or was not voluntary. To gain a better understanding of cannabinoid consumption for long-term pain management, we adapted an ad libitum, gelatin oral selfadministration paradigm [17-19] for the cannabinoids THC and CBD. Using this model in mice, we assessed long-term ( 3 week) consumption patterns, feeding microstructure and the long-term efficacy of these compounds in reducing chronic, neuropathic pain in comparison with orally consumed morphine.

\section{MATERIALS AND METHODS}

Animals

Male and female C57BL/6 mice $(n=143)$ ranging from 2 to 4 months of age were used in these experiments. All animals were

\footnotetext{
${ }^{1}$ Department of Pharmacology, University of Washington, 1959 NE Pacific St., Seattle, WA 98195, USA and ${ }^{2}$ Department of Psychiatry and Behavioral Sciences, University of Washington, 1959 NE Pacific St., Seattle, WA 98195, USA

Correspondence: Benjamin B. Land (BBL2@uw.edu)

These authors contributed equally: Antony D. Abraham, Edward J.Y. Leung
}

Received: 17 July 2019 Revised: 26 November 2019 Accepted: 28 November 2019

Published online: 7 December 2019 
singly housed under normal conditions (dark cycle beginning at 8:00 p.m. and ending at 6:00 a.m.), with standard chow and water ad libitum. All testing was performed from 1 to 4 p.m. All animals were drug naive with no prior procedures performed. Animal procedures were approved by the Institutional Animal Care and Use Committee of the University of Washington and conform to the guidelines on the care and use of animals by the National Institutes of Health.

\section{Drugs}

$\mathrm{THC}, \mathrm{CBD}$, and morphine were provided by the National Institute of Drug Abuse Drug Supply Program (Bethesda, MD). THC in ethanol $(\mathrm{EtOH})(100 \mathrm{mg} / \mathrm{ml})$ was diluted using $95 \% \mathrm{EtOH}$ to a stock concentration of $20 \mathrm{mg} / \mathrm{ml}$. CBD was dissolved into $95 \% \mathrm{EtOH}$ to a stock concentration of $20 \mathrm{mg} / \mathrm{ml}$. For i.p. injections, cannabinoids were first dissolved in $5 \% \mathrm{EtOH}$, then $5 \%$ cremophor was added, followed by sterile saline. Morphine was dissolved in sterile saline.

\section{Gelatin}

$3.85 \mathrm{~g}$ of Knox Gelatin was dissolved into $100 \mathrm{ml}$ of deionized $\mathrm{H}_{2} \mathrm{O}$ and raised to a temperature of $40^{\circ} \mathrm{C}$. Then either $5,2.5$, or $1 \mathrm{~g}$ of Polycal sugar was dissolved into the mixture to make the $5,2.5$, and $1 \%$ sugar content gels, respectively. While maintaining the temperature of the mixture between 41 and $43^{\circ} \mathrm{C}$, THC or CBD $(20 \mathrm{mg} / \mathrm{ml})$ was added to achieve a final drug concentration of $1 \mathrm{mg} / 15 \mathrm{ml}$ (g) of gelatin (or $0.5,2$, and $4 \mathrm{mg} / 15 \mathrm{ml}$ for THC). Morphine was added to achieve a concentration of $1.125 \mathrm{mg} /$ $15 \mathrm{ml}(\mathrm{g})$. Gelatin cooled in a $4^{\circ} \mathrm{C}$ refrigerator before use.

\section{Voluntary oral gelatin consumption}

Animals were singly housed, and a small plastic cup was secured to the metal wire top using a hose clamp. Approximately, $10 \mathrm{~g}$ of control gelatin (no drug) at 5\% Polycal content was inserted into each of the plastic cups at 2:00 p.m. for 3 days. The weight of the gelatin from the previous day was recorded and the remaining gelatin was discarded. After this, the Polycal content was reduced to $2.5 \%$ for 2 days, and again to $1 \%$ for 5 days. Two days prior to sciatic nerve ligation, gelatins were removed, and gelatin with drug was introduced 1 day after ligation, and every day thereafter until the study was concluded.

\section{Measurement of feeding microstructure}

A piezoelectric load cell was attached to a 3-D printed frame including the gelatin cup. The signal from the load cell was amplified and then sent to an Arduino mounted above the top of the cage for processing using custom code, and data was then stored on a flash storage device. Disturbances were defined as changes in mass greater than $0.04 \mathrm{~g}$ between recorded samples ( $2 \mathrm{~s}$ sample rate). A disturbance, or series of disturbances, was classified as a bout if there was a period greater than $120 \mathrm{~s}$ between consecutive disturbances. Schematics and all source code are available at GitHub: https://github.com/bwong07/ continuous-gel-self-administration-device.

Partial sciatic nerve ligation (pSNL)

pSNL was performed as previously described $[20,21]$ in Supplementary Materials and Methods.

Hot plate

A hot plate apparatus (IITC Life Science) was set to a temperature of $57.5^{\circ} \mathrm{C}$. Mice were gently placed onto the hot plate, and the latency of a pain response was recorded. A pain response was defined as either a paw lick, jump or a hind paw shuffle.

\section{Von Frey}

Von Frey hairs (IITC Life Science) of various, predetermined weights were used $(0.1-8 \mathrm{~g})$. Individual hairs of increasing weight (force) were pressed against each hind paw until a response (voluntary hind paw lift) was observed. The lowest weight that elicited a response for 2 out of 3 presses was recorded. We observed no differences in the ipsilateral and contralateral paw withdrawal scores $[22,23]$, so the two paws were averaged for each mouse.

\section{Locomotor behavior}

Locomotor activity was measured for $30 \mathrm{~min}$, both prior to and after drug administration. For a detailed description of analysis, see Supplementary Materials and Methods.

pSNL pain testing schedule

Mice were tested for hyperalgesia and allodynia (pain test) on the hot plate and von Frey, respectively. Baseline responses were measured 3 days prior, and immediately before surgery. A third pain test was administered $24 \mathrm{~h}$ after pSNL (day 1 post-pSNL), confirming a neuropathic pain state. Pain tests were then administered immediately prior to gelatin insertion at approximately 2:00 p.m. on days 5-, 7-, 12-, 15-, 18-, and 22-post pSNL. Pain data are expressed as percentage of baseline, which was calculated by averaging the two pre-pSNL pain test scores and dividing each subsequent pain test post-pSNL by that baseline value. Percentages below 100 indicate increases in pain.

\section{Acute pain testing schedule}

Mice were given control, THC, CBD, or morphine gelatins, as described above, for 7 days. On day 7 , an initial pain test was administered for all groups. On day 8, mice were placed in the locomotor chamber for 30-min, then initial rectal body temperatures were taken for control, THC, and CBD groups. Mice were then injected with vehicle, THC (10 or $30 \mathrm{mg} / \mathrm{kg}$ ), CBD (30 mg/kg), or morphine $(10 \mathrm{mg} / \mathrm{kg})$, corresponding to their drug gelatin. Following injection, mice were returned to locomotor chamber for 30-min, then pain tests were administered for morphine-treated mice. Two hour after control, THC, or CBD injection, rectal body temperatures were recorded, and pain tests were and administered. Drug-naïve animals went through the same procedure, with no gelatin exposure.

\section{Ultrasonic vocalizations}

A Petterson microphone (Norway, model M500-384, maximum recording frequency $=192 \mathrm{kHz}$ ) was inserted through a small hole in a filter top placed on top of the mouse's home cage. Fiveminute recordings were taken immediately before and after a pain test session using Avisoft SASLab Lite recording software. Recordings were analyzed using RavenLite (Cornell lab of Ornithology), by first generating a spectrogram of the recording and then manually scanning each file for ultrasonic broadband clicks, defined as an audio feature that met several criteria: (1) lasting less than $10 \mathrm{~ms},(2)$ having a minimum $10 \mathrm{KHz}$ frequency width, (3) having an average power density of greater than $-50 \mathrm{~dB}$ FS over $5 \mathrm{~ms}$ at its minimum frequency width, (4) having peak power in the ultrasonic range $(20-100 \mathrm{KHz})$, and (5) lacking a fundamental frequency or appreciable power below $15 \mathrm{kHz}$. These last two criteria were used to reject audible clicks. The number of clicks in each 5-min recording were quantified.

\section{Serum levels of drugs}

Animals were decapitated and trunk blood was collected, incubated at RT for 15-20 min, and spun for $10 \mathrm{~min}$ at $1800 \times \mathrm{g}$, at $4{ }^{\circ} \mathrm{C}$ for serum. Serum was stored at $-80^{\circ} \mathrm{C}$ and sent to the Mass Spectrometry Facility at University of Washington for processing against known standards for THC, CBD, and morphine [19].

\section{Data analysis}

All data were analyzed using MATLAB and GraphPad Prism 7-8. For all statistical testing (paired $t$ test, one- and two- way ANOVA, and post hoc analyses correcting for multiple comparisons), alpha level was set to 0.05 . 


\section{RESULTS}

Based on the gelatin free-access model developed by Clark and colleagues [17-19], we designed a paradigm to study long-term treatment outcomes of cannabinoid and opioid selfadministration in mice with chronic neuropathic pain (Fig. 1a).
After both sucrose fading and drug introduction, gelatin consumption was consistent within groups across the experiment (Fig. 1b), with no significant changes in consumption across days of access within any group (control gelatin $F_{4,180}=1.743, p=0.14$; drug gelatin $\left.F_{16,720}=1.342, p=0.16\right)$. Consumption of gelatin
A
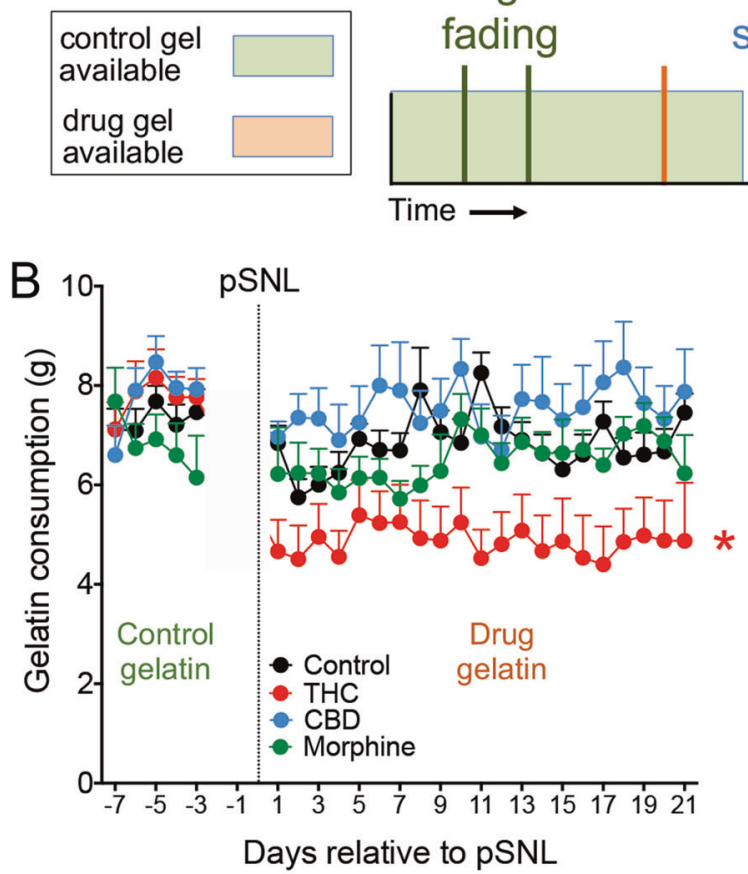

Drug available after $\mathrm{pSNL}$

$\mathrm{D}$

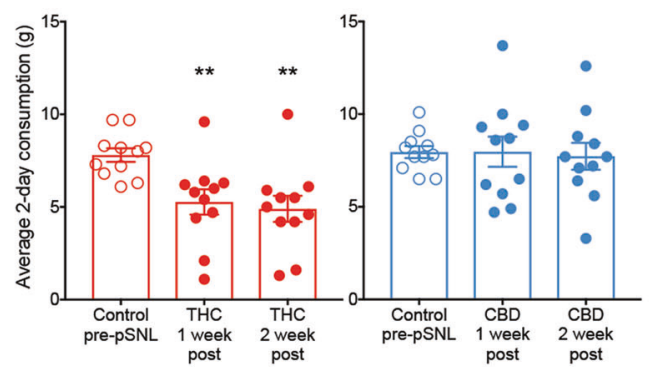

Drug available before and after pSNL

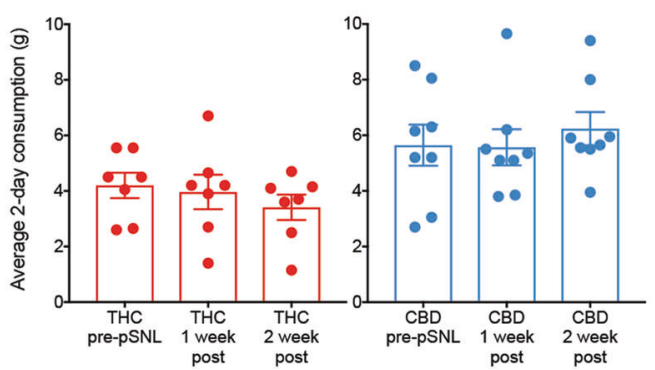

pSNL Pain Blood

surgery measures collection
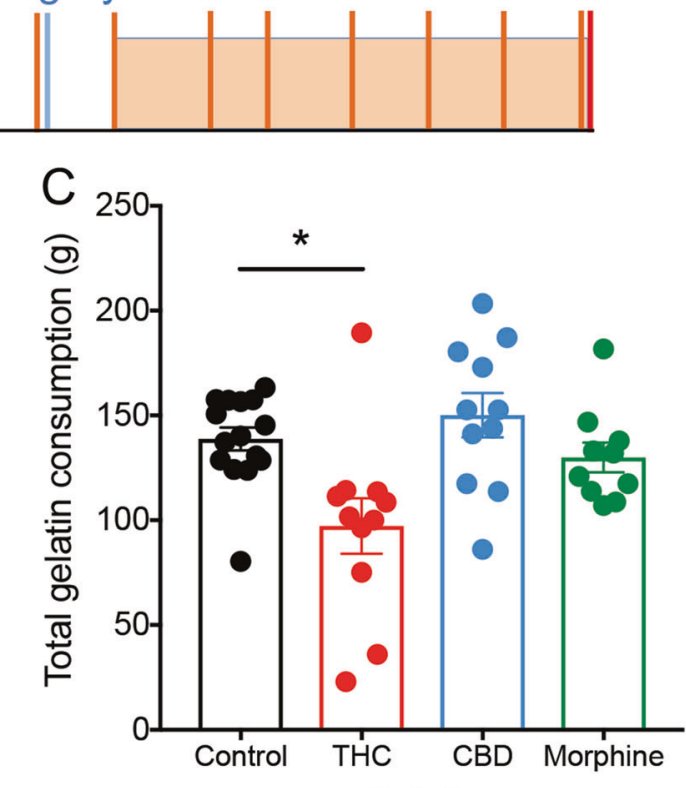

Gelatin
$E$

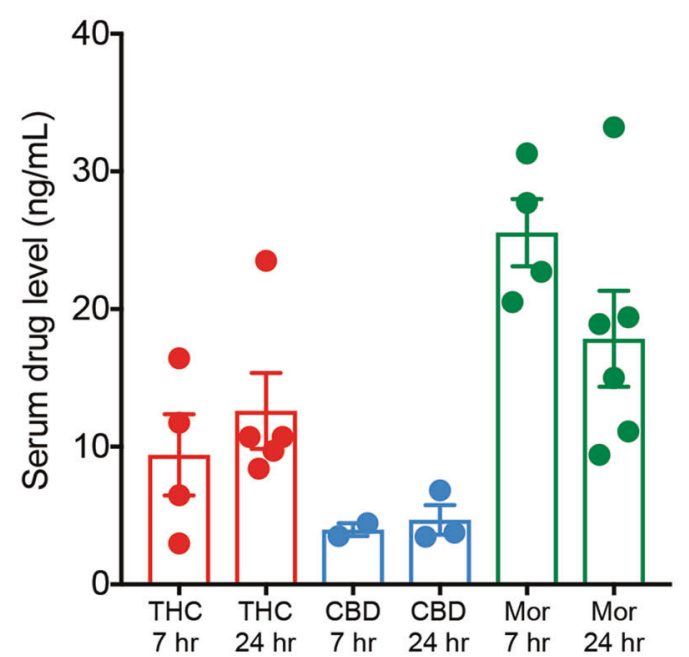

Fig. 1 Mice stably consume cannabinoid and opioid gelatin over 3 weeks. a Schematic of study design for chronic neuropathic pain and gelatin consumption. b Average daily consumption of gelatin for the 4 groups (control, THC, CBD, and morphine, $n=10-17$ ). Prior to pSNL, all groups consumed control gelatin. Mice consuming THC-gelatin ate significantly less than control mice $(p=0.003)$. c Total consumption of gelatin post-pSNL for control, THC, CBD, and morphine gelatins. Total THC-gelatin consumption was significantly lower than control mice ( $p=$ 0.005). d Comparisons within groups between the average consumption over the last 2 days prior to pSNL, days 6-7 after pSNL, and days 13-14 after pSNL. The top row represents animals who only received THC or CBD after pSNL. The bottom row represents animals that received THC or CBD before and after pSNL. Animals receiving drug gelatin prior to pSNL showed no differences in consumption after pSNL. e Serum levels of THC, CBD, and morphine collected either 7 or $24 \mathrm{~h}$ after the final gelatin insertion $(n=2-6) .{ }^{*} p<0.05,{ }^{* *} p<0.01$, data represent the mean \pm SEM. 
containing THC was significantly lower compared to control $\left(F_{3,45}=231, p=0.003\right.$, Dunnett's Control vs. THC $\left.p=0.012\right)$, and this effect was not observed in either the CBD or morphine gelatins. Similarly, total gelatin consumption over 3 weeks was significantly lower in THC-gelatin animals compared to control (Fig. 1c, 1-way ANOVA $F_{3,43}=5.92, p=0.0018$; Dunnett's Control vs. THC $p=0.005)$, whereas there was no difference from control in either the CBD or morphine groups. There was no apparent escalation of consumption following initial introduction of drug for any of the gelatins. To determine if the decrease in THC gelatin consumption was due to the presence of THC or the neuropathic pain state, a separate cohort of animals were trained to consume either THC or CBD gelatin both before and after pSNL (Fig. S1A). In both THC and CBD groups, the level of gelatin consumption after pSNL remained unchanged, indicating that occurrence of chronic pain does not alter consumption or produce escalated intake (Fig. 1d, THC $F_{1.45,8.72}=1.79, p=0.22 ; \mathrm{CBD} F_{1.65,11.55}=0.99$, $p=0.39$ ). We also determined if there was a dose-dependent relationship between gelatin THC concentration and consumption and found that mice consumed significantly less when the concentration was increased 4-fold, while reducing the concentration by half did not increase consumption to control levels (Fig. S1B, 1-way ANOVA $F_{3,20}=6.99, p=0.002$; Dunnett's $1 \mathrm{mg}$ vs. $4 \mathrm{mg} p=0.003)$. Interestingly, 20 days of consumption significantly increased weight gain in THC and CBD groups compared to control (Fig. S1C, 1-way ANOVA $F_{3,37}=6.57 p=0.001$; Holm-Sidak Control vs. THC $p=0.039$, Control vs. CBD $p=0.031$ ). To confirm that consumption of the drug gelatin produced circulating levels of drug in the mice, blood was collected either 7 or $24 \mathrm{~h}$ after the last gelatin insertion and serum was prepared. Detectable levels of all drugs were observed at every time point measured (Fig. 1e). Drug levels in THC-, CBD-, and morphine-consuming mice were similar between the 7 and $24 \mathrm{~h}$ time points, suggesting that animals maintain a relatively stable blood level of drug throughout the day. This prompted us to explore the patterns of drug consumption throughout the period of drug availability.

To enable a detailed analysis of drug consumption, we developed a measurement device that tracks the weight of the gelatin and any disturbances of the gelatin cup in real-time. The device consisted of a piezoelectric sensor (load cell) that measured changes in mass, a signal amplifier, and a microcontroller connected to a data storage device (Fig. 2a, Fig. S2A). Mouse interactions with the gelatin cup, which registered as brief and rapid changes in mass ("disturbances"), were recorded, as well as the overall, longer term changes in gelatin mass. A representative trace of both gelatin cup disturbances and cumulative gelatin consumed showed frequent interactions with gelatin cup and concordant increases in gelatin intake (Fig. 2b). This relationship was confirmed when we correlated the number of feeding bouts (defined as one or more disturbances separated from preceding or following disturbances by greater than $120 \mathrm{~s}$ ) with the amount of gelatin consumed in a 2 -h period (Fig. $2 c, r^{2}=0.44, F=98.5, p<$ $0.0001)$. Further, the accuracy of the piezoelectric sensor was independently confirmed by correlating the sensor-recorded mass with gel measurements weighed by experimenter on a standard scale over two time periods (Fig. S2B). We recorded consumption of all gelatins throughout the measurement period (Fig. 2d), and THC consumption was significantly lower than control by $18 \mathrm{~h}$, as in Fig. $1\left(F_{3,10}=5.70, p=0.015\right.$, Dunnett's Control vs. THC $p=$ $0.044)$. However, there were no significant differences between any of the groups in the number of feeding bouts throughout the day (Fig. 2e). This suggests that mice receiving THC gels were not feeding less frequently, but were consuming less during each bout. Gelatin consumption followed expected circadian patterns, and consumption was approximately 2-fold higher during the dark phase when the animals were awake and more active (Fig. S2C, Time $F_{1,10}=43.04, p<0.001$, Holm Sidak Dark cycle vs. Light cycle $p<0.05)$.
Once we confirmed that mice were stably consuming gelatin, we tested to see if this level of consumption was sufficient to produce significant relief of chronic neuropathic pain. The pSNL surgery produced allodynia, a painful response to a normally nonpainful stimulus, as measured by Von Frey hairs in mice after 1 day (Fig. 3a). This allodynia persisted throughout the experiment in animals that only received control gelatin, and both THC and CBD significantly relieved allodynia compared to control mice (Fig. 3a, Interaction $F_{15,235}=3.07, p<0.001$, Dunnett's post hoc). This effect was apparent on the first pain test after drug-gelatin presentation (day 5) and was maintained throughout the 3 weeks of testing, with a modest decrease in THC efficacy on the final two testing days. Morphine relieved allodynia maximally on the sixth day following initial introduction of drug (day 7), but on day 12 post pSNL paw withdrawal response thresholds decreased to similar levels as control animals. Examining only the final day of testing, mice that received either THC or CBD gelatin had significantly reduced allodynia compared to mice that received control gelatin (Fig. 3b, $F_{3,47}=5.885, p=0.002$, Holm-Sidak Control vs. THC $p=0.049$, Control vs. CBD $p=0.002$ ). There was no difference between mice that received morphine or control gelatin. In contrast to the effects of cannabinoids on allodynia, the hyperalgesia (an increased pain response to a normally painful stimuli) measured by hot plate took longer to develop following pSNL and was more variable in all groups (Fig. 3c). In the first week of drug gelatin exposure, morphine produced a significant analgesic effect that disappeared by day 12 post $\mathrm{pSNL}$, while neither THC or CBD produced any significant effect (Interaction $F_{15,160}=1.76, p=0.044$, Dunnett's post hoc). Examining only the final day of testing (Fig. 3d), THC showed a significant reduction in hyperalgesia compared to controls, while there was a trend effect of $\mathrm{CBD}$ and no effect of morphine $\left(F_{3,32}=3.2, p=0.036\right.$, Control vs. THC $p=0.02$, Holm-Sidak Control vs. CBD $p=0.093$, Control vs. Morphine $p=0.35$ ).

To study the analgesic responses in a neuropathic pain-naïve state and to confirm the development of morphine tolerance, we examined behavioral effects of experimenter-administered drug in drug-naive and drug-exposed animals with 7 days of gelatin selfadministration. Consumption of either THC or CBD gelatin for 7 days did not change basal measurements of body temperature, locomotor activity, analgesia (hot plate response), or allodynia (Von Frey) from mice receiving control gelatin (Fig. S3A-D). Mice that consumed morphine gelatin also showed no significant differences from control gelatin for locomotor activity, analgesia, or allodynia (Fig. S3B-D). We then tested whether prior gelatin consumption produced tolerance by injecting the same mice with their corresponding drugs and comparing their behavioral scores with drug-naïve mice receiving a matching injection. As expected, THC injection (30 mg/kg i.p.) produced a significant decrease in body temperature, decreased locomotor activity, increased paw withdrawal threshold, and increased hot plate analgesia compared to controls (Fig. $4 a-d$, body temperature interaction $F_{2,18}=$ 4.72, $p=0.023$, Holm Sidak Control vs. THC $p<0.001$; locomotor $F_{3,23}=3.29, p=0.039$, Holm Sidak Control vs. THC $p=0.042$; Von Frey $F_{3,23}=6.63, p=0.002$, Holm Sidak Control vs. THC $p=0.006$; hot plate $F_{3,28}=3.20, p=0.038$; Holm Sidak Control vs. THC $p<$ 0.001). There were no significant differences between 7-day gelatin and drug-naïve animals on any measure for THC, and we observed similar overall effects at a lower magnitude with a 10 $\mathrm{mg} / \mathrm{kg}$ dose of THC, although body temperature showed a trend towards tolerance in the 7-day group (Fig. S4, $t$ test $t_{9}=2.06, p=$ 0.07). In contrast, CBD injection did not affect the behavioral measurements compared to control or drug-naïve mice. This was surprising and suggests that CBD may only produce an analgesic effect if pain is already established (Fig. 4a-d). Finally, morphine injection in drug-naïve mice produced significant increases in locomotor activity, analgesia and paw withdrawal threshold (Fig. 4b-d, Holm Sidak drug-naïve, locomotor $p=0.005$, Von Frey 
A
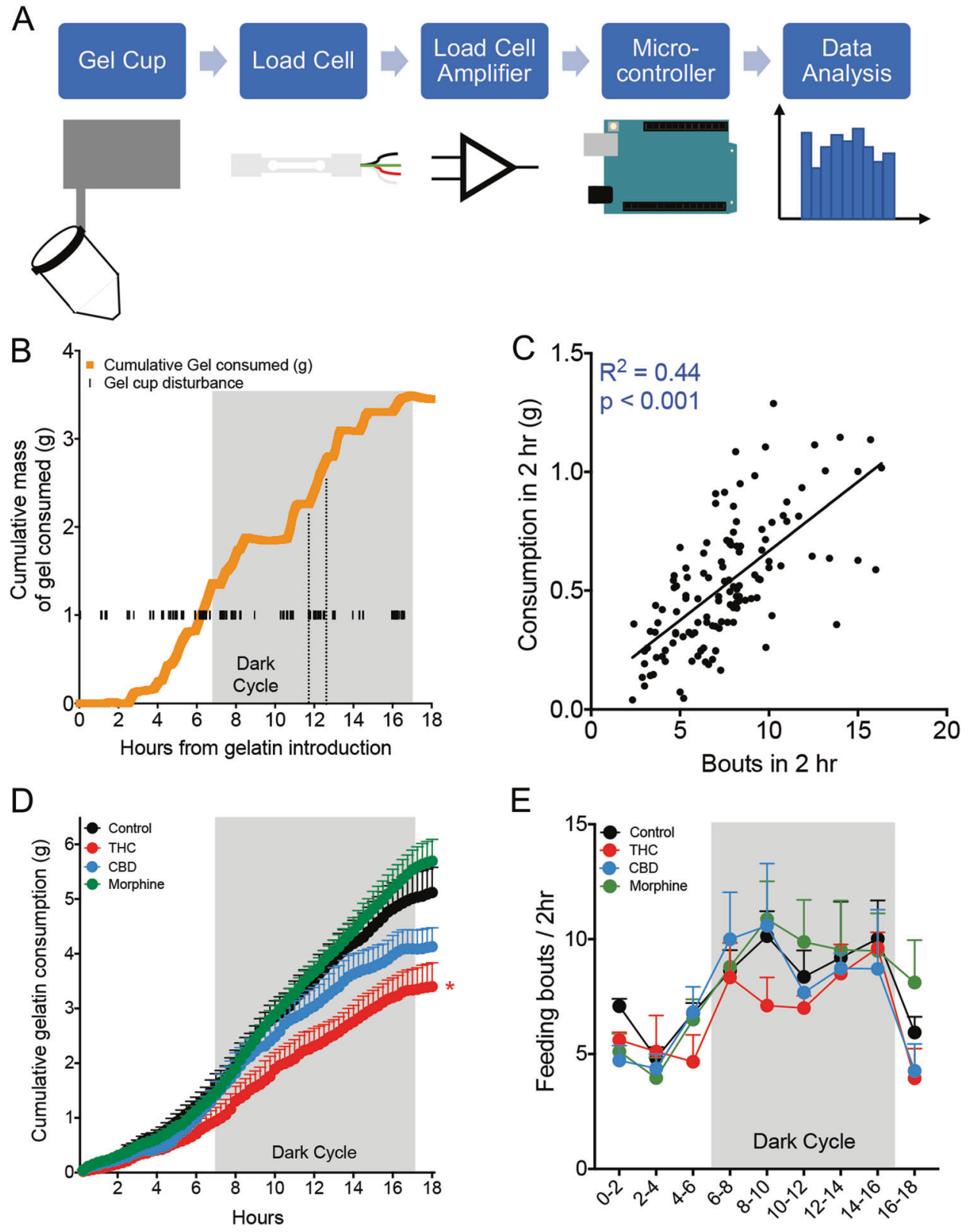

E

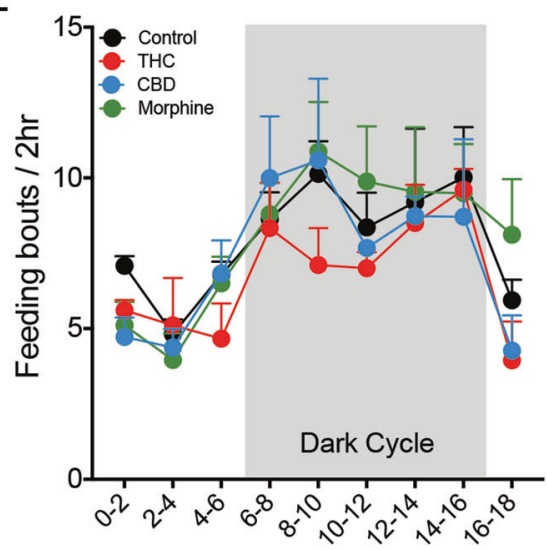

Hour from gelatin introduction

Fig. 2 Microstructure of gelatin consumption. a Schematic and signal flow of piezoelectric load cell that measures second-by-second consumption and disturbances of the gelatin cup. $\mathbf{b}$ Example of cumulative gelatin consumption and disturbances of the gelatin cup over $18 \mathrm{~h}$ from one mouse. $\mathrm{c}$ Correlation between the number of disturbances of the gelatin cup and the consumption of gelatin in 5 min windows $(n=126$ points from 14 animals, $p<0.001)$. d Average cumulative consumption of the control, THC, CBD, and morphine gelatins over the $18 \mathrm{~h}$ measurement period. By $18 \mathrm{~h}$, THC mice showed significantly less cumulative consumption than controls $(n=3-4$ mice per group, $p=0.04)$. e Average number of bouts, defined as a set of disturbances preceded or succeeded by at least 2 min, during $2 \mathrm{~h}$ windows throughout the measurement period. There were no differences between the groups in the number of bouts $\left(n=3-4\right.$ mice per group). ${ }^{*} p<0.05$, data represent the mean \pm SEM.

Control vs. Morphine $p<0.001$, hot plate Control vs. Morphine $p=0.007)$. However, these effects were not present in animals that had consumed morphine gelatin for 7 days, confirming the previous observation that mice had developed tolerance to morphine (Holm Sidak Morphine naïve vs. 7-day, locomotor $p<$ 0.003 , Von Frey $p<0.001$, hot plate $p=0.037$ ). These results demonstrate that week-long oral consumption of THC, in contrast to morphine, did not produce tolerance.

Because measurements of pain in mice often require a subjective scoring metric, we explored whether mice exhibited different types of vocalizations as a function of chronic neuropathic pain and subsequent treatment. Using a wide frequency range microphone (up to $192 \mathrm{KHz}$, Petterson), we recorded ultrasonic vocalizations of mice in their home cages 5 min before pain testing on 4 days in control, THC, CBD, and morphine gelatin groups. In these singly-housed mice, we did not observe many traditional ultrasonic vocalizations, such as rising or falling calls. However, we did notice the presence of many ultrasonic, broadband clicks, an understudied mouse vocalization hypothesized to be a negative con-specific signal 


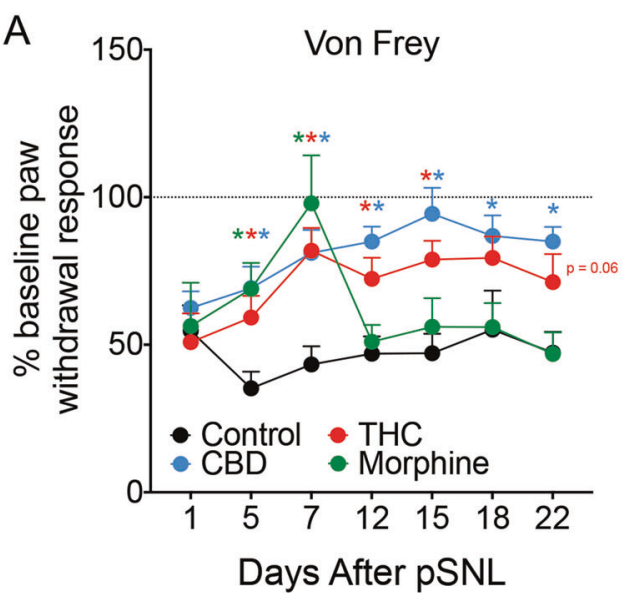

C

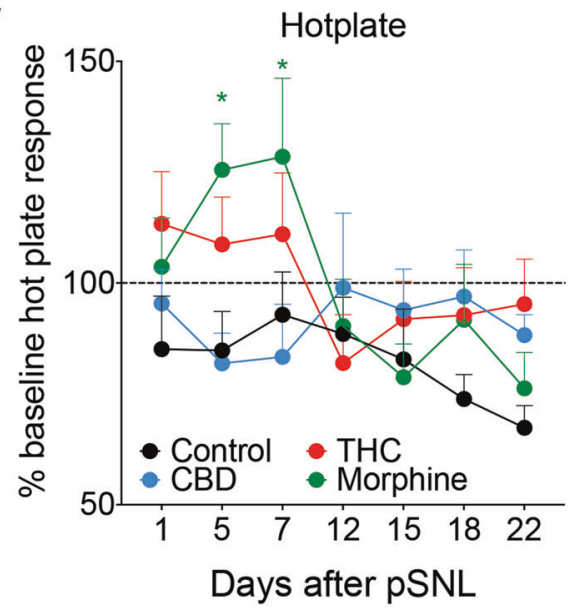

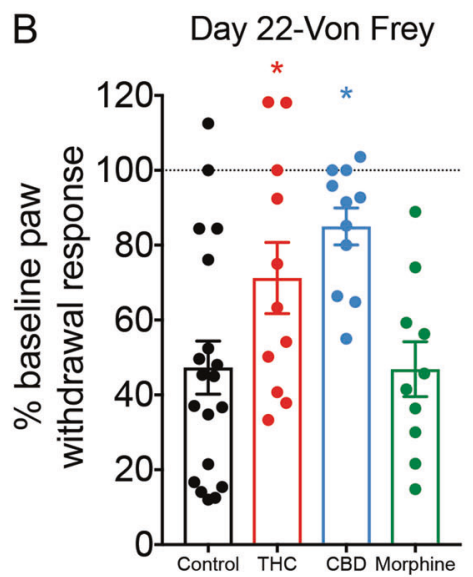

Gelatin

$\mathrm{D}$

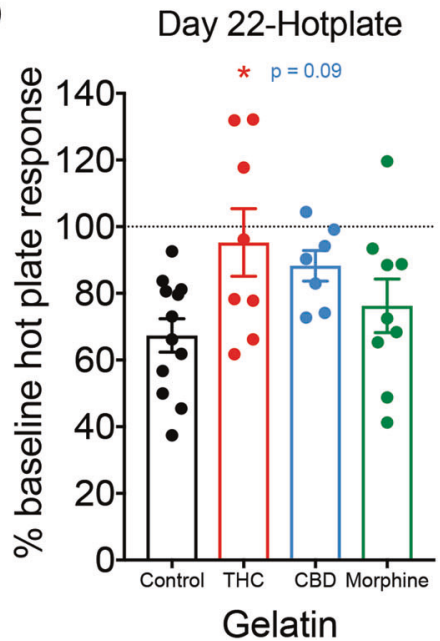

Fig. 3 Cannabinoid consumption decreases measurements of neuropathic pain over 3 weeks. a Paw withdrawal thresholds, plotted as a percentage of pre-pSNL response, are shown 1 day after pSNL, and on the 6 subsequent testing days after introduction of either control, THC, CBD, or morphine gelatin. THC and CBD groups showed long-lasting reduction in allodynia, while the effect of morphine lasted approximately 1 week ( $n=10-19$ per group). b Comparison of percentage of pre-pSNL response on day 22, the final day of pain testing, between the 4 treatment groups ( $n=10-19$ per group). c Latency to respond to hot plate, plotted as a percentage of pre-pSNL response, are shown 1 day after pSNL, and on the 6 subsequent testing days after introduction of either control, THC, CBD, or morphine gelatin. The morphine group showed an analgesic response in the first week, while no other groups were significantly different from control ( $n=7-12$ per group). d Comparison of percentage of pre-pSNL response on day 22. THC significantly reduced hyperalgesia while there was a trend effect of CBD ( $n=7-12$ per group). ${ }^{*} p<0.05$, data represent the mean \pm SEM.

[24] (Fig. 5a, Fig. S5A). In a neuropathic pain-free state, mice showed an increase in ultrasonic clicks after pain tests were administered (Fig. S5B, paired $t$ test $t_{5}=2.64, p=0.046$ ). These clicks were not attributable to other environmental stimuli, as empty cage recordings had very few clicks (Fig. S5B). Compared to their neuropathic pain-free state, mice increased click vocalizations following pSNL surgery (Fig. $5 \mathrm{~b}$, paired $t$ test $\left.\mathrm{t}_{15}=6.14, p<0.001\right)$. Furthermore, 4-days after initial introduction of either THC, CBD, or morphine gelatin, click vocalizations were significantly reduced compared to control gelatin, and were comparable to baseline counts prior to pSNL surgery (Fig. $5 b, F_{2,13}=14.46, p<0.001$, Sidak Control vs. THC $p=0.001$, Control vs. CBD $p=0.002$, Control vs. Morphine $p=0.006$ ). Because of individual variability in click vocalizations prior to the introduction of drug, these data were separated by treatment group and analyzed using within-subject statistical tests (Fig. 5c). Although there were differences in the magnitude of response (driven largely by the variance in clicks after neuropathic pain), the effect of THC, CBD, or morphine in reducing the number of clicks remained significant (Fig. 5c, all groups $p<0.05$, Tukey's post hoc $p<0.05)$.

\section{DISCUSSION}

The principle findings of this study are that (1) animals will voluntarily consume both cannabinoids and opioids in a gelatin preparation, (2) these doses are sufficient to produce analgesia over short (morphine) and long (THC and CBD) time periods without dose escalation, and (3) ultrasonic clicks may be an ethological and objective representation of pain status. These findings indicate that both THC and CBD may be suitable for longterm treatment of chronic pain, in contrast to morphine. CBD may be especially beneficial in treatment of chronic pain because it lacks the rewarding psychoactive effects of THC and does not produce analgesia in a pain-naïve state (Fig. 4), while still providing significant relief of allodynia during chronic pain. Hyperalgesia and allodynia reflect different environmental stimuli, and while predictable painful stimuli can often be avoided, allodynic responses to stimuli like normal touch cannot. Therefore, the long-term inhibition of allodynia by CBD, without tolerance or psychoactive side-effects, could be a future indication along with its established role in seizure prevention [25]. However, it is important to note that we did see an increase in weight in the CBD groups, implying that one adverse side effect of chronic 
A
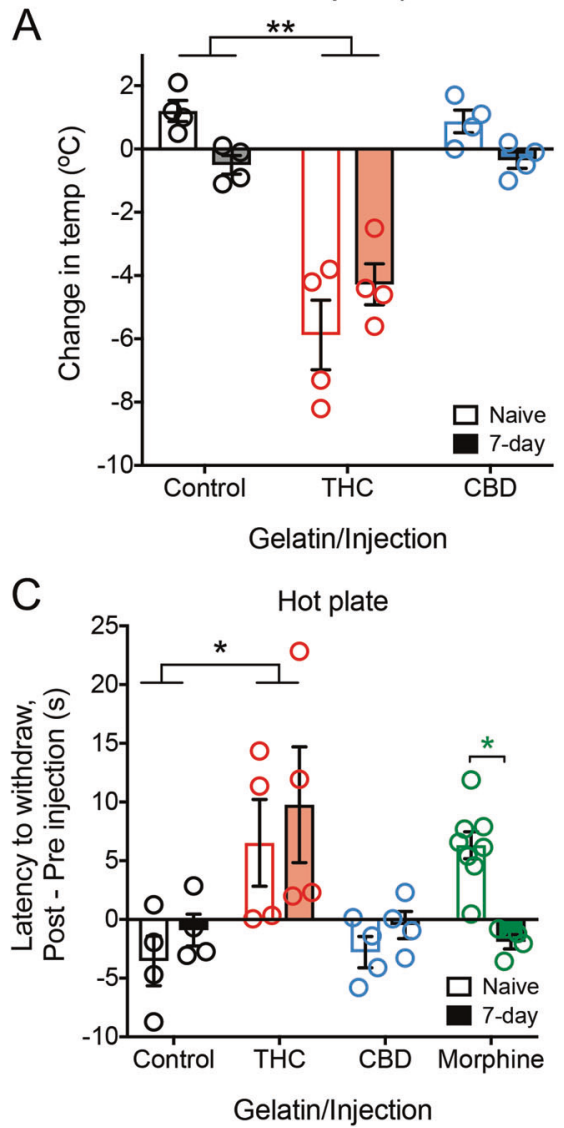
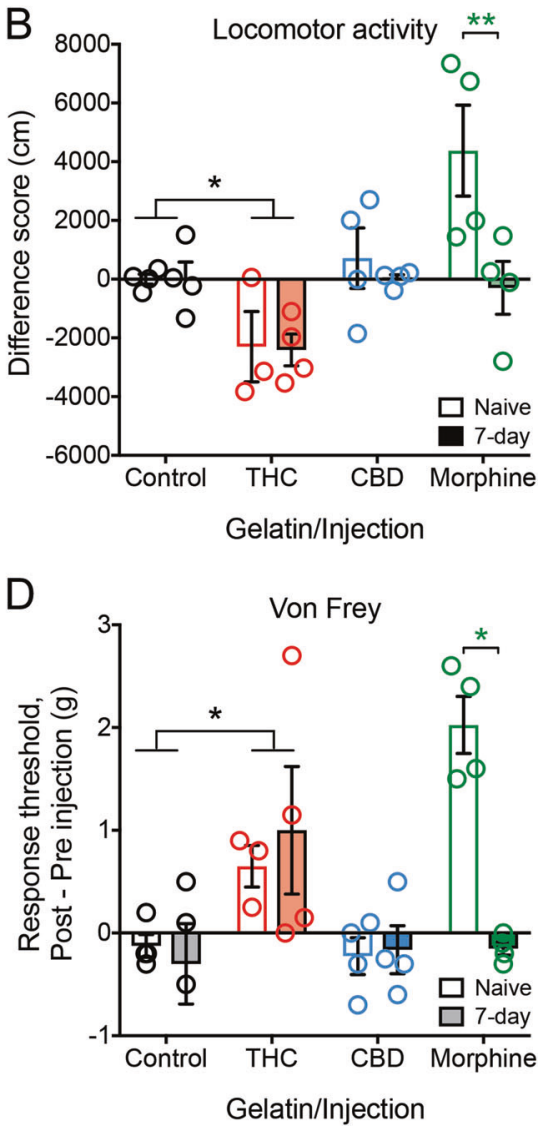

Fig. 4 Measures of analgesia and body temperature in neuropathic pain-naïve mice. a Change in body temperature $2 \mathrm{~h}$ after either vehicle, THC (30 mg/kg i.p.), or CBD (30 mg/kg i.p.) experimenter-administered dose in 7-day gelatin consuming animals or drug-naïve controls. THC injected mice showed the predicted decrease in body temperature in both groups, whereas CBD showed no change ( $n=4$ per group). b Change in locomotor activity after either vehicle, THC (30 mg/kg i.p.), CBD (30 mg/kg i.p.), or morphine (10 mg/kg i.p.) experimenteradministered dose in 7-day gelatin consuming animals or drug-naïve controls ( $n=3-4$ per group). c Change in latency to pain response $2 \mathrm{~h}$ after either vehicle, THC (30 mg/kg i.p.), CBD (30 mg/kg i.p.), or $30 \mathrm{~min}$ after morphine $(10 \mathrm{mg} / \mathrm{kg}$ i.p.) experimenter-administered dose in 7 -day gelatin consuming animals or drug-naïve controls. THC injected mice in both groups showed a similar increase in analgesic response, while CBD animals showed no change. Drug-naïve morphine injected animals showed analgesia, while there was no change in mice that have consumed morphine for 7 days ( $n=4-8$ per group). $\mathbf{d}$ Change in paw withdrawal threshold $2 \mathrm{~h}$ after either vehicle, THC (30 mg/kg i.p.), CBD (30 mg/kg i.p.), or $30 \mathrm{~min}$ after morphine (10 mg/kg i.p.) experimenter-administered dose in 7-day gelatin consuming animals or drug-naïve controls. THC injected mice in both groups showed a similar increase in paw withdrawal threshold, while CBD animals again showed no change. Drug-naïve morphine injected animals showed an increase in paw withdrawal threshold, while there was no change in mice that have consumed morphine for 7 days ( $n=3-4$ per group). ${ }^{*} p<0.05,{ }^{* *} p<0.01$, data represent the mean \pm SEM.

CBD consumption could be weight gain. Why CBD fails to produce analgesia in pain-naïve mice is unknown, but may be related to its established anti-inflammatory role [26-28], and that pro-inflammatory pain signals are required for CBD to be effective.

Oral gelatin-based models of self-administration offer potential benefits over existing methods for cannabinoid and opioid intake. Theoretically, animals would be able to control their drug dose by increasing or decreasing consumption with a negligible caloric component. For example, in the THC gelatin-consuming group, animals ate significantly less gelatin than controls throughout the study, and we verified that as THC concentrations increased further, there was a corresponding decrease in consumption (Figure S1B). This decrease in intake could be due to the psychoactive effects of THC, or a potentially aversive olfactory or gustatory feature of the THC gelatin. The current experimental design cannot distinguish between these possibilities, but this may not be important in the context of THC-dependent pain relief. In contrast, we did not observe greater consumption of morphinegelatin compared to control, suggesting that other features of the gelatin such as texture or sugar content may drive consumption. Subsequent studies using other drugs of abuse and/or eliminating sugar will help determine if the positive reinforcing qualities of drugs promotes consumption in this gelatin model.

Another benefit of this paradigm is that animals remain in their home cage, minimizing stress of different environments and allowing normal consumption of chow and water, in addition to gelatin. Oral consumption is a popular ingestion method for cannabis products, as it does not require vapor inhalation which could be aversive or ill-suited to certain populations (e.g., lung cancer patients), and drug effects last longer due to the pharmacokinetics of oral administration [29]. We suggest that our model provides a reliable and closer preclinical representation of a major form of cannabis intake in humans. The blood concentration of orally consumed THC in this study matches concentrations $(5-10 \mathrm{ng} / \mathrm{mL})$ that have been observed to produce significant impairments in cognitive and motor performance in humans [30]. Further experiments are required to determine whether chronic THC consumption produces significant cognitive or motivational impairment in mice. The consumed doses of THC over $24 \mathrm{~h}$ are likely similar to experimenter-administered doses that produce anxiety-related behaviors [31, 32] and analgesic effects [33], but direct comparison between these routes of 

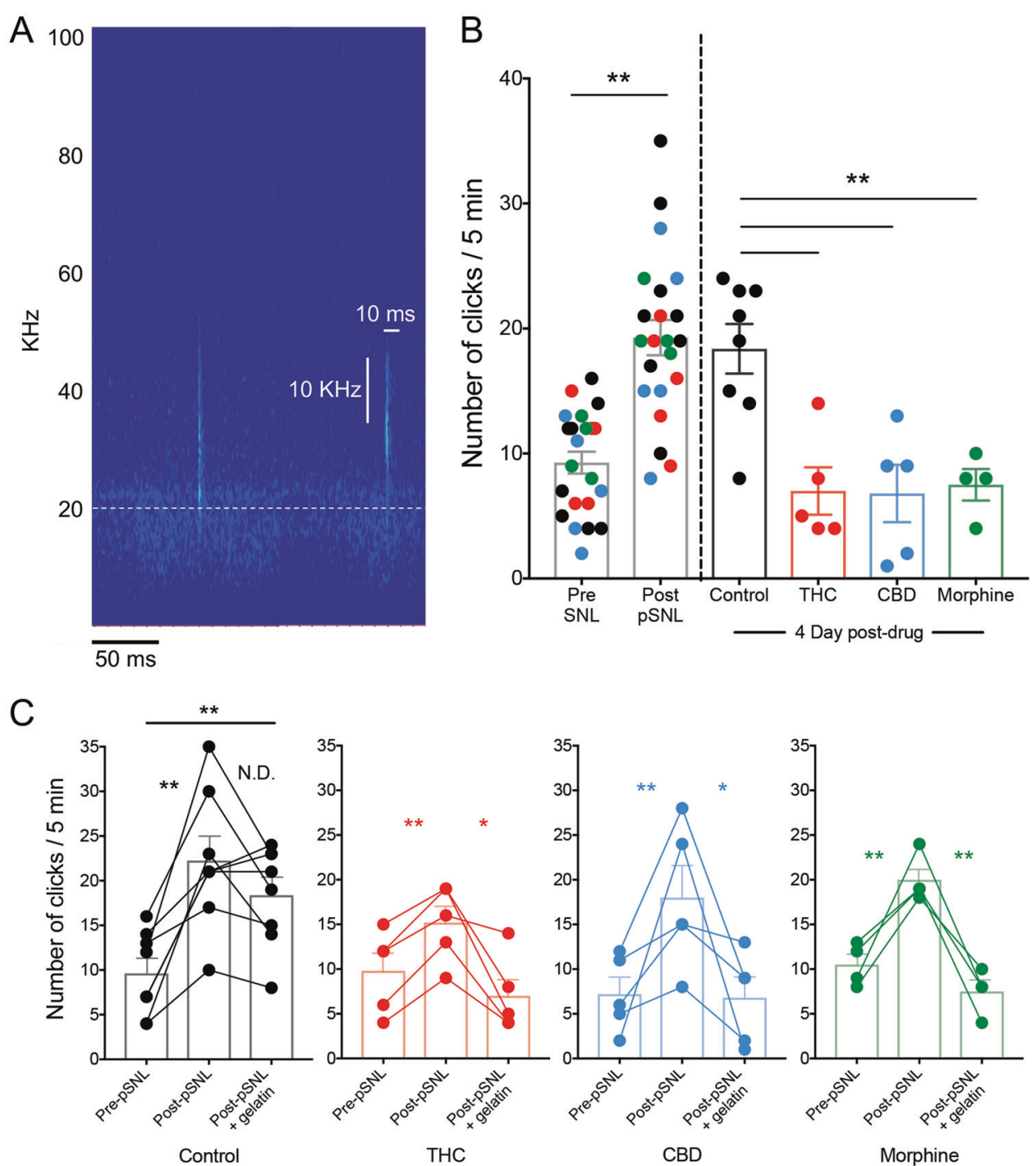

Fig. 5 Ultrasonic click vocalizations track pain state. a Two representative ultrasonic clicks. Each is under $10 \mathrm{ms,} \mathrm{wider} \mathrm{than} 10 \mathrm{kHz}$, with no appreciable power in the audible range. Dashed line represents $20 \mathrm{kHz}$, the upper limit of the human audible range. $\mathbf{b}$ Number of ultrasonic clicks per 5 min recording interval. Colors represent control (black), THC (red), CBD (blue), and morphine (green) gelatin mice ( $n=4-7$ per group). pSNL significantly increased the number of clicks $(p<0.001)$. After 4 days of gelatin, the number of clicks remained elevated in control mice, while THC, CBD, and morphine mice showed decreases similar to baseline counts. c Separated clicks for each treatment group. All groups showed a significant increase in clicks after $\mathrm{pSNL}$, and in THC, CBD, and morphine consuming mice clicks significantly decreased after drug-gelatin consumption. ${ }^{*} p<0.05,{ }^{* *} p<0.01$, data represent the mean \pm SEM.

administration is difficult due to differences in pharmacokinetics and dynamics.

It is important to note that we did not observe escalation in this model with morphine gelatin, even when tolerance developed, which we would predict from a human taking morphine for pain [34]. It is possible that escalation requires periods of drug abstinence, thus escalation may occur more readily in a paradigm with limited access. However, little evidence was seen for THCgelatin escalation in rats under various limited-access schedules [19]. Further testing with opioids that have been shown to produce escalation in self-administration paradigms, like fentanyl or heroin [35], may help to determine if escalation is possible with free-access gelatin.

The current study did not identify the molecular mechanism of cannabinoid-mediated long-term pain relief. The cannabinoid receptors (CB1R, CB2R) themselves are likely candidates, as they have both been implicated in antinociception [36-38]. However, previous studies have shown that the analgesic effects of cannabinoids can occur in CB1R or CB2R knockout mice, implying an alternative target $[28,39]$. These targets are diverse and include transient receptor potential (TRP) channels [40], other G proteincoupled receptors [41,42], and glycine receptors [28, 43]. We hope to identify the neural substrates and mechanisms of action using this paradigm in the future.

We developed a device to measure real-time changes in gelatin mass. The piezoelectric load cell enabled recording of both the rapid changes in mass associated with animal contact, and cumulative changes in gelatin mass. By using this system, we verified both the lower intake of THC that we first observed with our daily gelatin measurements, and an increase in consumption during the dark cycle compared to the light. The components for the load cell measurement system are affordable and this technology should be relatively easy to adapt to other consumption media.

The inclusion of ultrasonic clicks in our study was intended to capture an objective, ethological feature of the pain response; one 
that did not require an experimenter-determined score. The observation that neuropathic pain produced increases in ultrasonic clicks, reversible by cannabinoids and morphine, suggests these clicks may represent either a painful or a general aversive state, which has been previously described [24]. Whether a click carries relevant con-specific information or is an epiphenomenon of some other process, remains unknown. One hypothesis is that a brief, broad spectrum click would be harder to audibly triangulate than a long call, and so mice could use clicks as a way of communicating a dangerous, aversive situation, without alerting nearby predators. Further ethological validation of this vocalization is warranted.

In conclusion, we have demonstrated that a gelatin selfadministration paradigm allows voluntary consumption of drug over long periods and may be useful as an alternative to classical operant behavioral designs for drug intake. In addition, we have shown that mice produce click vocalizations that track pain status, which can be recorded non-invasively and may provide an objective correlate of pain. Finally, we have shown that the cannabinoids THC and CBD, in contrast to morphine, produce long-lasting relief of chronic neuropathic pain in a sciatic nerve injury model in mice. Specifically, CBD may represent a viable therapeutic option because of its low psychoactive profile, lack of efficacy in a pain-free state, and long-lasting reduction in allodynia in chronic pain.

\section{FUNDING AND DISCLOSURE}

These studies were funded by SCAN Design Foundation (BBL), the University of Washington Alcohol and Drug Abuse Institute (ADAl, Small Grants Program, BBL), and DA030074 (Charles Chavkin). The authors declare no competing interests.

\section{ACKNOWLEDGEMENTS}

We thank Sanne Casello for assistance with surgery, Dr. Charles Chavkin for experimental support, Dr. Nephi Stella for critical review of the paper, and the Mass Spectrometry facility at the University of Washington for serum processing.

\section{ADDITIONAL INFORMATION}

Supplementary Information accompanies this paper at (https://doi.org/10.1038/ s41386-019-0585-3)

Publisher's note Springer Nature remains neutral with regard to jurisdictional claims in published maps and institutional affiliations.

\section{REFERENCES}

1. Johannes CB, Le TK, Zhou X, Johnston JA, Dworkin RH. The prevalence of chronic pain in United States adults: results of an internet-based survey. J Pain. 2010; 11:1230-9.

2. Committee on Advancing Pain Research, Care, and Education, Institute of Medicine, Board on Health Sciences Policy. Relieving Pain in America: A Blueprint for Transforming Prevention, Care, Education, and Research. Washington, DC: The National Academies Press; 2011.

3. Pitcher MH, Korff MV, Bushnell MC, Porter L. Prevalence and profile of highimpact chronic pain in the United States. J Pain. 2019;20:146-60.

4. Gaskin DJ, Richard P. Institute of Medicine (US) Committee on Advancing Pain Research, Care, and Education. Relieving Pain in America: A Blueprint for Transforming Prevention, Care, Education, and Research. Appendix C: The Economic Costs of Pain in the United States. US: National Academies Press; 2017.

5. Williams JT, Ingram SL, Henderson G, Chavkin C, von Zastrow M, Schulz S, et al. Regulation of $\mu$-opioid receptors: desensitization, phosphorylation, internalization, and tolerance. Pharmacol Rev. 2013;65:223-54.

6. Schattauer SS, Land BB, Reichard KL, Abraham AD, Burgeno LM, Kuhar JR, et al. Peroxiredoxin 6 mediates Gai protein-coupled receptor inactivation by cJun kinase. Nat Commun. 2017;8:743.

7. Fields HL, Margolis EB. Understanding opioid reward. Trends Neurosci. 2015;38: 217-25.
8. Phillips JK, Ford MA, Bonnie RJ, National Academies of Sciences, Engineering, and Medicine; Health and Medicine Division; Board on Health Sciences Policy; Committee on Pain Management and Regulatory Strategies to Address Prescription Opioid Abuse. Pain Management and the Opioid Epidemic: Balancing Societal and Individual Benefits and Risks of Prescription Opioid Use. US: National Academies Press; 2017.

9. Manzanares J, Julian M, Carrascosa A. Role of the cannabinoid system in pain control and therapeutic implications for the management of acute and chronic pain episodes. Curr Neuropharmacol. 2006;4:239-57.

10. Elikottil J, Gupta P, Gupta K. The analgesic potential of cannabinoids. J Opioid Manag. 2009;5:341-57.

11. Vučković S, Srebro D, Vujović KS, Vučetić Č, Prostran M. Cannabinoids and pain: new insights from old molecules. Front Pharmacol. 2018;9:1259.

12. Romero-Sandoval EA, Kolano AL, Alvarado-Vázquez PA. Cannabis and cannabinoids for chronic pain. Curr Rheumatol Rep. 2018;19:67.

13. Lötsch J, Weyer-Menkhoff I, Tegeder I. Current evidence of cannabinoid-based analgesia obtained in preclinical and human experimental settings. Eur J Pain. 2018;22:471-84.

14. Nguyen JD, Aarde SM, Vandewater SA, Grant Y, Stouffer DG, Parsons LH, et al. Inhaled delivery of $\Delta(9)$-tetrahydrocannabinol (THC) to rats by e-cigarette vapor technology. Neuropharmacology. 2016;109:112-20.

15. Javadi-Paydar M, Nguyen JD, Kerr TM, Grant Y, Vandewater SA, Cole M, et al. Effects of $\triangle 9-T H C$ and cannabidiol vapor inhalation in male and female rats. Psychopharmacology (Berl). 2018;235:2541-57.

16. Barrus DG, Lefever TW, Wiley JL. Evaluation of reinforcing and aversive effects of voluntary delta-9 tetrahydrocannabinol ingestion in rats. Neuropharmacology. 2018;137:133-40.

17. Schindler AG, Tsutsui KT, Clark JJ. Chronic alcohol intake during adolescence, but not adulthood, promotes persistent deficits in risk-based decision-making Alcohol Clin Exp Res. 2014;38:1622-9.

18. Nasrallah NA, Clark JJ, Collins AL, Akers CA, Phillips PE, Bernstein IL. Risk preference following adolescent alcohol use is associated with corrupted encoding of costs but not rewards by mesolimbic dopamine. Proc Natl Acad Sci USA. 2011;108:5466-71.

19. Kruse LC, Cao JK, Viray K, Stella N, Clark JJ. Voluntary oral consumption of $\Delta^{9}$ tetrahydrocannabinol by adolescent rats impairs reward-predictive cue behaviors in adulthood. Neuropsychopharmacology. 2019;44:1406-14.

20. Seltzer Z, Dubner R, Shir Y. A novel behavioral model of neuropathic pain disorders produced in rats by partial sciatic nerve injury. Pain. 1990; 43:205-18.

21. Xu M, Petraschka M, McLaughlin JP, Westenbroek R, Caron MG, Lefkowitz RJ, et al. Neuropathic pain activates the endogenous kappa opioid system in mouse spinal cord and induces opioid receptor tolerance. J Neurosci. 2004;24:4576-84.

22. Koltzenburg M, Wall PD, McMahon SB. Does the right side know what the left is doing? Trends Neurosci. 1999;22:122-7.

23. Arguis MJ, Perez J, Martinez G, Ubre M, Gomar C. Contralateral neuropathic pain following a surgical model of unilateral nerve injury in rats. Reg Anesth Pain Med. 2008;33:211-6.

24. Sugimoto H, Okabe S, Kato M, Koshida N, Shiroishi T, Mogi K, et al. A role for strain differences in waveforms of ultrasonic vocalizations during male-female interaction. PLoS ONE. 2011;6:e22093.

25. Kaplan JS, Stella N, Catterall WA, Westenbroek RE. Cannabidiol attenuates seizures and social deficits in a mouse model of Dravet syndrome. Proc Natl Acad Sci USA. 2017;114:11229-34.

26. Li H, Kong W, Chambers CR, Yu D, Ganea D, Tuma RF, et al. The non-psychoactive phytocannabinoid cannabidiol (CBD) attenuates pro-inflammatory mediators, $T$ cell infiltration, and thermal sensitivity following spinal cord injury in mice. Cell Immunol. 2018;329:1-9.

27. Mukhopadhyay $P$, Rajesh M, Horváth B, Bátkai S, Park O, Tanchian G, et al. Cannabidiol protects against hepatic ischemia/reperfusion injury by attenuating inflammatory signaling and response, oxidative/nitrative stress, and cell death. Free Radic Biol Med. 2011;50:1368-81.

28. Xiong W, Cui T, Cheng K, Yang F, Chen SR, Willenbring D, et al. Cannabinoids suppress inflammatory and neuropathic pain by targeting a3 glycine receptors. J Exp Med. 2012;209:1121-34

29. Ohlsson A, Lindgren JE, Wahlen A, Agurell $S$, Hollister $L E$, Gillespie HK. Plasma delta-9 tetrahydrocannabinol concentrations and clinical effects after oral and intravenous administration and smoking. Clin Pharmacol Ther. 1980; 28:409-16.

30. Ramaekers JG, Moeller MR, van Ruitenbeek P, Theunissen EL, Schneider E, Kauert G. Cognition and motor control as a function of Delta9-THC concentration in serum and oral fluid: limits of impairment. Drug Alcohol Depend. 2006;85:114-22.

31. Berrendero F, Maldonado R. Involement of the opioid system in the anxiolytic-like effects induced by Delta9-tetrahydrocannabinol. Psychopharmacology (Berl). 2002;163:111-7. 
32. Kasten CR, Zhang Y, Boehm SL II. Acute cannaninoids produce robust anxiety-like and locomotor effects in mice, but long-term consequences are age- and sexdependent. Front Behav Neurosci. 2019;13:32.

33. Casey SL, Atwal N, Vaughan CW. Cannabis constituent synergy in a mouse neuropathic pain model. Pain. 2017;158:2452-60.

34. Zernig G, Ahmed SH, Cardinal RN, Morgan D, Acquas E, Foltin RW, et al. Explaining the escalation of drug use in substance dependence: models and appropriate animal laboratory tests. Pharmacology. 2007;80:65-119.

35. Wade CL, Vendruscolo LF, Schlosburg JE, Hernandez DO, Koob GF. Compulsivelike responding for opioid analgesics in rats with extended access. Neuropsychopharmacology. 2015;40:421-8.

36. Mulpuri Y, Marty VN, Munier JJ, Mackie K, Schmidt BL, Seltzman HH, et al. Synthetic peripherally-restricted cannabinoid suppresses chemotherapy-induced peripheral neuropathy pain symptoms by CB1 receptor activation. Neuropharmacology. 2018;139:85-97.

37. Hanus L, Breuer A, Tchilibon S, Shiloah S, Goldenberg D, Horowitz M, et al. HU308: A specific agonist for $\mathrm{CB}(2)$, a peripheral cannabinoid receptor. Proc Natl Acad Sci USA. 1999;96:14228-33.
38. Malan TP Jr, Ibrahim MM, Lai J, Vanderah TW, Makriyannis A, Porreca F. CB2 cannabinoid receptor agonists: pain relief without psychoactive effects? Curr Opin Pharmacol. 2003;3:62-7.

39. Zimmer A, Zimmer AM, Hohmann AG, Herkenham M, Bonner TI. Increased mortality, hypoactivity, and hypoalgesia in cannabinoid $C B 1$ receptor knockout mice. Proc Natl Acad Sci USA. 1999;96:5780-85.

40. Muller C, Morales $\mathrm{P}$, Reggio PH. Cannabinoid ligands targeting TRP channels. Front Mol Neurosci. 2019;11:487.

41. Rodríguez-Muñoz M, Onetti $Y$, Cortés-Montero E, Garzón J, Sánchez-Blázquez P. Cannabidiol enhances morphine antinociception, diminishes NMDA-mediated seizures and reduces stroke damage via the sigma 1 receptor. Mol Brain. 2018;11:51.

42. Guerrero-Alba R, Barragán-Iglesias $P$, González-Hernández A, Valdez-Moráles EE, Granados-Soto $V$, Condés-Lara $M$, et al. Some prospective alternatives for treating pain: the endocannabinoid system and its putative receptors GPR18 and GPR55. Front Pharmacol. 2019;9:1496.

43. Xiong W, Cheng K, Cui T, Godlewski G, Rice KC, Xu Y, et al. Cannabinoid potentiation of glycine receptors contributes to cannabis-induced analgesia. Nat Chem Biol. 2011;7:296-303. 